\title{
ENFERMAGEM DE REABILITAÇÃO NA TRANSIÇÃO DA PESSOA COM ALTERAÇÃO MOTORA POR AVC: REVISÃO SISTEMÁTICA DA LITERATURA
}

\section{ENFERMERÍA DE REHABILITACIÓN EN LA TRANSICIÓN DE LA PERSONA CON ALTERACIONES MOTORAS POR ACV: REVISIÓN SISTEMÁTICA DE LA LITERATURA}

REHABILITATION NURSING IN THE TRANSITION OF THE PERSON WITH MOTOR DISABILITY AFTER CVA: SYSTEMATIC LITERATURE REVIEW

DOI 10.33194/rper.2020.v3.n2.2.5770 | Submetido 25/04/2020 | Aprovado 15/12/2020

\author{
Maria de Fátima Godinho de Matos $^{1}$; ; Joaquim Augusto Gonçalves Simões² \\ 1 - Centro Hospitalar Médio Tejo; 2 - Escola Superior de Saúde do Instituto Politécnico de Santarém
}

\section{RESUMO}

Introdução: 0 acidente vascular cerebral (AVC) tem um impacto enorme na vida das pessoas e famílias. Frequentemente, a pessoa em situação de AVC apresenta alterações motoras que carecem da intervenção fulcral do enfermeiro especialista em Enfermagem de Reabilitação (EEER) na implementação de um programa de reabilitação, integrado na equipa interdisciplinar, para garantir uma transição segura para o domicílio.

Objetivo: Identificar as intervenções do EEER que capacitam a pessoa e família/cuidador, em situação de AVC com alteração motora na preparação do regresso casa.

Método: Revisão sistemática da literatura nas bases de dados Pub Med, EBSCO e ProQuest, com aplicação de critérios de inclusão e exclusão, num horizonte temporal de 5 anos (2014-2019).

Resultados: Foram incluídos 3 artigos que reforçam a necessidade da aplicação de programas de reabilitação que integrem as dimensões física, psicológica e cognitiva da pessoa, bem como o papel fundamental dos cuidadores familiares na gestão da dependência e das atividades de vida diárias (AVD) da pessoa com AVC.

Conclusão: A intervenção do EEER deve estar integrada num programa de reabilitação motora, com recurso a estratégias: educação para a saúde; identificação e capacitação do cuidador; ensino e treino de AVD e referenciação para os recursos da comunidade, com o objetivo de promover o treino e supervisão, dando continuidade ao processo de reabilitação.

Palavras-chave: AVC; enfermagem; movimento; reabilitação

\section{RESUMEN}

Introducción: El accidente cerebrovascular (ACV) tiene un gran impacto en la vida de la persona y su familia. Con frecuencia, la persona en situación de ACV presenta alteraciones motoras, las cuales carecen de la imprescindible intervención de la enfermera especialista en Enfermería de Rehabilitación (EEER) en la implementación de un programa de rehabilitación, integrado en el equipo multidisciplinar, para garantizar una transición segura al hogar.

Objetivo: Identificar las intervenciones de la EEER que empoderan a la persona y familia / cuidador, en situación de ACV con alteración motora en la preparación para el regreso a casa.

Método: Revisión sistemática de la literatura en las bases de datos Pub Med, EBSCO y ProQuest, con aplicación de criterios de inclusión y exclusión, en un horizonte temporal de 5 años (2014-2019).

Resultados: Se incluyeron 3 artículos que refuerzan la necesidad de la aplicación de programas de rehabilitación que integren las dimensiones física, psicológica y cognitiva de la persona, así como los cuidadores familiares en el manejo de la dependencia en las actividades de la vida diaria (AVD) de la persona con ACV.

Conclusión: La intervención EEER debe integrarse en un programa de rehabilitación motora, utilizando estrategias: educación para la salud; identificación y capacitación del cuidador; Enseñanza y capacitación en las AVD y derivación a recursos comunitarios con el fin de promover la capacitación y supervisión, continuando el proceso de rehabilitación.

Palabras clave: ACV; enfermería; movimiento; rehabilitación

\section{ABSTRACT}

Introduction: Cerebral vascular accident (CVA) has a huge impact on a person's life and his family. Frequently, the person with stroke presents motor alterations, which need the essential intervention of the specialistized 
rehabilitation nurse (SRN) in order to implement a rehabilitation program, integrated in an interdisciplinary team, to ensure a safe return home.

Objective: To identify the SRN interventions that empower the person with motor disability after CVA and his family/caregiver, in the preparation for the return home.

Method: Systematic literature review in the Pub Med, EBSCO and ProQuest databases, with application of inclusion and exclusion criteria, over a 5-year time horizon (2014-2019).

Results: The 3 articles included reinforced the need for the application of rehabilitation programs that integrate the person 's physical, psychological and cognitive dimensions, as well as the family caregivers in the management of the disability and daily life activities (DLA) in the person with CVA.

Conclusion: The SRN intervention must be integrated into a motor rehabilitation program, using the strategies: health education, identification and training of the caregiver, DLA teaching and training and referral to community resources in order to promote training and supervision, ensuring rehabilitation process continuity.

Keywords: CVA; nursing; movement; rehabilitation

\section{INTRODUÇÃO}

Ao longo da vida são vários os processos e transições que a pessoa e família/cuidador vão experienciando, nomeadamente o diagnóstico da doença, alteração na dependência, alta hospitalar e, paralelamente, uma constante redefinição de papéis familiares. Assim, o enfermeiro especialista em Enfermagem de Reabilitação (EEER), de acordo com a suas competências, elabora, implementa, monitoriza e avalia programas de reeducação funcional e de treino, capacitando a pessoa para o autocontrolo e autocuidado (1). Neste contexto, o EEER assume um papel imprescindível para um processo de transição saudável e eficaz ${ }^{(2)}$.

$O$ acidente vascular cerebral (AVC) além de ser primeira causa de morte em Portugal, apresenta elevada morbilidade e impacto social, tornando-se evidente a necessidade do desenvolvimento e implementação de estratégias interdisciplinares e intersectoriais, no âmbito da prevenção, tratamento e reabilitação precoce ${ }^{(3)}$.

O tratamento do AVC agudo envolve o internamento hospitalar, com segurança e qualidade, com o objetivo de recapacitar e reintegrar a pessoa no seu ambiente familiar e, tanto quanto possível, no local de trabalho, através da intervenção precoce, direcionada à neurorreabilitação, identificação de fatores de risco, prevenção de complicações, tratamento de comorbilidades e desenvolvendo um plano de alta e de follow up adequados ${ }^{(4)}$.

A hospitalização, principalmente na pessoa idosa, tem influência a nível da capacidade funcional e da capacidade de realização do autocuidado, devido à imobilidade e respetivas complicações ${ }^{(5)}$. Assim, a intervenção com vista à reabilitação deve ser interdisciplinar e o mais precoce possível, de modo a manter e recuperar as funções físicas, psicológicas e sociais, contribuindo para a prevenção de complicações, nomeadamente, úlceras de pressão, trombose venosa profunda, embolia pulmonar e incapacidade funcional (6) (7).

0 regresso a casa é um indicador de reabilitação com sucesso e, consequentemente, um indicador de qualidade. Deste modo, o planeamento da alta iniciase no momento da admissão, na qual a equipa numa perspetiva interdisciplinar realiza uma avaliação e compreensão dos fatores psicológicos ${ }^{(8)}$, $\operatorname{cognitivos}^{(9)} \mathrm{e}$ físicos que influenciam o regresso ao domicílio. A avaliação da pessoa deve envolver aspetos pessoais e do funcionamento quotidiano, contribuindo para a criação de uma relação terapêutica, com definição de medidas realistas, através da realização do exame neurológico, aplicação de escalas de avaliação apropriadas e gestão eficiente dos recursos (5) (6) (3).

Para que a pessoa possa ser independente ou atingir o máximo de funcionalidade possível é necessário o treino das capacidades remanescentes e/ou uso de estratégias adaptativas, como os produtos de apoio. 0 EEER, de acordo com as suas competências específicas (1), seleciona e prescreve produtos de apoio e realiza treinos específicos de atividades de vida diárias (AVD), promovendo o ensino e supervisão da utilização dos mesmos, tendo em vista a máxima capacidade funcional da pessoa, em segurança, prevenindo o risco de queda. A prevenção de quedas é um indicador de qualidade e, como tal, o EEER juntamente com a equipa interdisciplinar deve integrar no programa de reabilitação um conjunto de atividades com vista à maximização da funcionalidade com segurança, tendo em conta os fatores intrínsecos e extrínsecos ${ }^{(6)}$ (7) (10).

A educação para a saúde contribui também para a diminuição do reinternamento em unidades de cuidados agudos. Assim, o EEER deve recorrer a estratégias como as reuniões e sessões de educação para a saúde, no âmbito da prevenção da doença, através dos fatores de risco, educação específica para a doença, ao nível da fisiologia, plano de tratamento e reabilitação e autogestão, envolvendo a resolução de problemas, tomada de decisão, utilização de recursos e criação de planos de ação ${ }^{(11)}$.

Numa situação de doença, os papéis familiares sofrem alterações. Maioritariamente, o cônjuge (quando existe) assume o papel de prestador de cuidados e/ou novos papéis em casa, o que pode criar stress pelo sentimento de obrigação e de conflito. Neste sentido, o EEER é fundamental para assegurar a continuidade de cuidados, promovendo a inclusão da pessoa e família no processo de cuidados e reabilitação, onde os membros da família devem desenvolver autocapacitação e estratégias de coping, que são essenciais para transições saudáveis ${ }^{(11)}$. 
Deste modo, o programa de reabilitação deve ser baseado nos padrões de exercício que contribuem para a conservação da força muscular, tendo em conta a pessoa e a sua motivação, o tipo de ação/contração muscular presente, a intensidade, o número de repetições, o tempo de repouso entre séries, a ordem sequencial dos exercícios, a sua frequência e ainda a compreensão de fenómenos fisiológicos e de neuroplasticidade ${ }^{(12)}$. As mobilizaçõos precoces, treino de equilíbrio ${ }^{(8)}$, treino ergonómico, treino de marcha, caminhada ao ar livre, treino em grupo, exercícios orientados pelo cuidador, posicionamento inibidor de reflexos, automobilizações, treino com produtos de apoio são exemplos chave no sucesso da reabilitação motora ${ }^{(13)}$.

Assim, face ao exposto um programa de reabilitação motor deve ser confluente, organizado e sintetizado, tornando-se importante identificar as intervenções de Enfermagem de Reabilitação que capacitam a pessoa com AVC, com alteração motora, bem como a sua família/cuidador, garantindo uma transição segura para o domicílio.

\section{METODOLOGIA}

Do ensino clínico à experiência profissional surgem várias questões, sendo emergente a necessidade de promover uma prestação de cuidados baseada na evidência científica. Seguindo o percurso desenvolvido pelos autores em 2018 em "A importância da Enfermagem de Reabilitação na preparação do regresso a casa da pessoa e família/cuidador em situação de AVC com alteração da funcionalidade motora"(14), foi realizada uma nova revisão sistemática da literatura tendo por base um protocolo de pesquisa com o objetivo de responder à questão PICo (P: participantes, I: intervenções, Co: contexto): (I) Que intervenções de Enfermagem de Reabilitação capacitam $(P)$ a pessoa $e$ família/cuidador, em situação de AVC, com alteração da funcionalidade a nível motor, na (Co) preparação do regresso a casa?

Os descritores de pesquisa e respetivo Scope Note foram definidos e validados na plataforma MeSH Brower - 2019, os quais constituem descritores MeSH para a pesquisa. Assim, foram inseridos nas bases de dados os descritores Nurs* (AND) Rehabilitation (AND) Stroke (AND) Movement, pela respetiva ordem apresentada, e com a expressão AND. Tendo por base a questão PICo foram definidos os critério de inclusão e exclusão que podem ser observados no quadro 1.

A pesquisa foi elaborada nas bases de dados científicas: PubMed, CINAHL Complete, MEDLINE Complete, Nursing \& Allied Health Collection: Comprehensive e ProQuest Nursing \& Allied Health Source, com aplicação de limitadores nas diferentes bases de dados, tendo em conta os critérios de inclusão e exclusão e limitadores de pesquisa, como é visível no quadro 2.

Num horizonte temporal de 5 anos (2014-2019), foram identificados 105 artigos. Após a aplicação dos critérios de inclusão e exclusão pela leitura de título e resumo, através da metodologia PRISMA, foram excluídos 6 por estarem repetidos e 96 por não cumprirem todos os critérios de inclusão, tendo sido selecionados 3 artigos para a revisão sistemática.

Os artigos incluídos na revisão sistemática da literatura, de natureza qualitativa e quantitativa foram submetidos a análise do conteúdo, respeitando os critérios de qualidade metodológica. A avaliação dos títulos, dos resumos (abstracts) e dos artigos identificados, foi realizada por dois pesquisadores, de forma independente, obedecendo aos critérios de inclusão e exclusão definidos no protocolo de pesquisa, verificando-se convergência entre os mesmos.

Quadro 1: Critérios de inclusão e exclusão

\begin{tabular}{|c|c|c|}
\hline & Critérios inclusão & Critérios exclusão \\
\hline $\begin{array}{l}\text { Tipo de } \\
\text { Participantes }\end{array}$ & $\begin{array}{l}\text { Pessoas com idade } \\
\text { superior a } 19 \text { anos. } \\
\text { Pessoas em situação de } \\
\text { AVC. }\end{array}$ & $\begin{array}{l}\text { Pessoas com idade inferior } \\
\text { a } 19 \text { anos. } \\
\text { Pessoas com alterações } \\
\text { motoras, condicionadas } \\
\text { por outras etiologias que } \\
\text { não AVC. }\end{array}$ \\
\hline & $\begin{array}{l}\text { Intervenções de Enf. } \\
\text { de Reabilitação que } \\
\text { incluam a: }\end{array}$ & $\begin{array}{l}\text { Intervenções de } \\
\text { Enfermagem de } \\
\text { reabilitação que não }\end{array}$ \\
\hline $\begin{array}{l}\text { intervenção/ } \\
\text { Fenómeno } \\
\text { de interesse }\end{array}$ & $\begin{array}{l}\text { - Capacitação da } \\
\text { pessoa e } \\
\text { família/cuidador, }\end{array}$ & $\begin{array}{l}\text { incluam as } 3 \text { condições: } \\
\text { - Capacitação da pessoa e } \\
\text { família/cuidador, }\end{array}$ \\
\hline $\begin{array}{l}\text { Tipos de } \\
\text { resultados }\end{array}$ & $\begin{array}{l}\text { - Reabilitação da } \\
\text { funcionalidade motora } \\
\text { e da mobilidade, } \\
\text { - Programação do } \\
\text { regresso ao domicílio. }\end{array}$ & $\begin{array}{l}\text { - Reabilitação da } \\
\text { funcionalidade motora e } \\
\text { da mobilidade, } \\
\text { - Programação do regresso } \\
\text { ao domicílio. }\end{array}$ \\
\hline $\begin{array}{l}\text { Tipos de } \\
\text { estudo }\end{array}$ & $\begin{array}{l}\text { Estudos quantitativos e } \\
\text { qualitativos, num } \\
\text { horizonte temporal de } \\
5 \text { anos (2014-2019). }\end{array}$ & $\begin{array}{l}\text { Excluem-se revisões } \\
\text { sistemáticas da literatura. }\end{array}$ \\
\hline
\end{tabular}

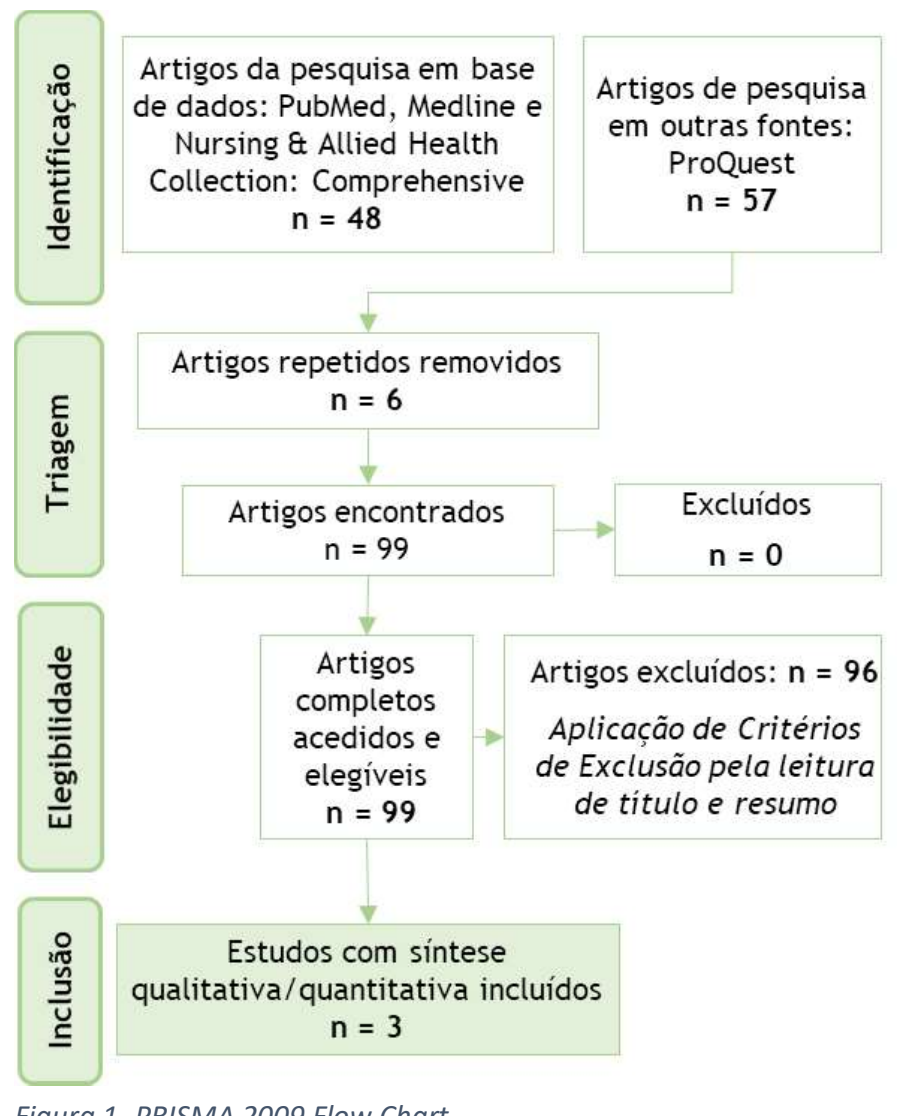

Figura 1- PRISMA 2009 Flow Chart 


\begin{tabular}{|c|c|c|c|c|c|}
\hline & \multirow[b]{2}{*}{ Pub Med } & \multicolumn{3}{|c|}{ EBSCO } & ProQuest \\
\hline & & CINAHL & MEDLINE & $\begin{array}{l}\text { Nursing \& } \\
\text { Allied Health } \\
\text { Collection: } \\
\text { Comprehensive }\end{array}$ & ProQuest Nursing \& Allied Health Source \\
\hline $\begin{array}{l}\text { Limitadores de } \\
\text { pesquisa, } \\
\text { selecionados } \\
\text { de acordo com } \\
\text { os critérios de } \\
\text { inclusão e } \\
\text { exclusão } \\
\text { definidos } \\
\text { previamente }\end{array}$ & $\begin{array}{l}\text { Abstract } \\
\text { Free Full } \\
\text { Text } \\
\text { Publicação } \\
\text { dos últimos } 5 \\
\text { anos } \\
\text { Humanos } \\
\text { Língua } \\
\text { inglesa } \\
\text { Faixa etária: } \\
\text { mais de } 19 \\
\text { anos }\end{array}$ & $\begin{array}{l}\text { Booleano } \\
\text { Língua Inglês } \\
\text { Texto completo } \\
\text { Resumo disponível } \\
\text { Qualquer autor: } \\
\text { enfermeiro } \\
\text { Idioma: inglês } \\
\text { Humanos } \\
\text { Faixa etária: mais de } \\
19 \text { anos } \\
\text { Data da publicação: } \\
1 \text { de junho de } 2014 \text { a } \\
30 \text { de junho de } 2019\end{array}$ & $\begin{array}{l}\text { Booleano } \\
\text { Texto completo } \\
\text { Língua inglesa } \\
\text { Resumo disponível } \\
\text { Idade relacionada } \\
\text { com: mais de } 19 \text { anos } \\
\text { Humanos } \\
\text { Data da publicação: } 1 \\
\text { de junho de } 2014 \text { a } 30 \\
\text { de junho de } 2019\end{array}$ & $\begin{array}{l}\text { Booleano } \\
\text { Texto completo } \\
\text { Resumo } \\
\text { disponível } \\
\text { Data da } \\
\text { publicação: } 1 \text { de } \\
\text { junho de } 2014 \text { a } \\
30 \text { de junho de } \\
2019 \text {. }\end{array}$ & $\begin{array}{l}\text { Data: } 1 \text { de junho2014 a } 30 \text { junho de } 2019 \\
\text { Faixa etária: Adulto (19-44 anos), Idoso (+65 } \\
\text { anos), Idoso (+80 anos), Meia-idade (45-64 } \\
\text { anos) } \\
\text { Tipo de fonte: Revistas, Revistas Académicas, } \\
\text { Revistas especializadas } \\
\text { Tipo de documento: Artigo, Artigo Principal, } \\
\text { Crítica, Crítica literária, Discurso/Palestra, } \\
\text { Dissertação/Tese, Documento de referência, } \\
\text { Estudo de caso, Livro, Medicina baseada em } \\
\text { evidências, Primeira página/Artigo da capa, } \\
\text { Relatório, Relatório anual, Relatório técnico } \\
\text { Idioma: Inglês } \\
\text { Assunto Principal - Rehabilitation }\end{array}$ \\
\hline
\end{tabular}

Pesquisas realizadas em Santarém no mês de julho de 2019.

\section{RESULTADOS}

O recurso à revisão sistemática permitiu a análise de artigos com conteúdo científico atualizado e com validade, numa perspetiva de enquadramento da temática trabalhada, revelando-se assim num contributo fundamental na sustentação e validação da temática em estudo.

O primeiro estudo incluído ${ }^{(8)}$, com nível de evidência 3.c (15), foi desenvolvido na Austrália em 2016, com a participação 134 pessoas com AVC residentes na comunidade, recrutadas no período de internamento da doença. 0 estudo tinha como objetivo a elaboração de um questionário Stroke Exercise Preference Inventory (SEPI), através da aplicação de escalas, utilização de dados demográficos e dados sobre o evento de doença, que permitisse avaliar as preferências no exercício e barreiras, na pessoa após AVC e ainda determinar a relação entre as caraterísticas pessoais e determinar a relação entre as características pessoas (incapacidade, fadiga, depressão, ansiedade) e as preferências e barreiras no exercício, para avaliar se as características podem influenciar as diferenças individuas no SEPI. Para tal, a elaboração do SEPI foi desenvolvido primeiramente em duas etapas, uma de desenvolvimento e outra de aperfeiçoamento do conteúdo, do qual resultou o SEPI-35 e, posteriormente, surgiu a versão final SEPI 13, com a categorização de sete fatores de preferência do exercício: apoio e supervisão (presença de instrutor, profissional), desafio e confiança, bem-estar/saúde, contexto do exercício (junto de familiares, amigos, período do dia, em casa, na rua), estar sozinho em casa, outros similares (grupos comunitários, pessoas com AVC, idade semelhante, mesmo género) e música-TV (durante os exercícios ouvem música ou vêm TV).

Os autores (8) concluíram que existem múltiplas dimensões nas preferências e condições do exercício, destacando a necessidade de exercícios pós-AVC sob aplicação de programas. Para algumas pessoas com mais incapacidade, receber supervisão e apoio profissional pode gerar sentimentos de controle durante exercício e reduzir o medo relacionado com a segurança. A insegurança está relacionada com a incapacidade funcional, depressão e fadiga, sugerindo que os programas de exercício devem para além de envolver a dimensão física, devem envolver a dimensão psicológica. Pessoas com sintomas depressivos apresentaram níveis baixos de confiança e dão preferências à música e TV, como estratégias para minimizar a dor e desconforto. As pessoas com sintomas de ansiedade demonstraram vontade de realizar os exercícios com outras pessoas com a mesma condição física, idade e género, diminuído o risco de avaliação negativa. No entanto, para outros a supervisão e o apoio servem apenas para desenvolver o desejo de independência e autonomia ${ }^{(11)}$.

O instrumento SEPI permitiu avaliar as preferências e barreiras no exercício nas pessoas com AVC, no domicílio, podendo ser aplicado por profissionais de saúde, sem treino prévio, permitindo analisar e compreender a relação entre preferências individuais de exercícios e adesão a programas de exercícios após o AVC ${ }^{(8)}$.

O segundo estudo ${ }^{(16)}$, desenvolvido no Irão em 2017, com nível de evidência 3.c (15), tem como objetivo explorar e descrever as experiências dos cuidadores familiares sobre as estratégias para lidar com o idoso com AVC dependente nas AVD.

Os dados foram recolhidos através de entrevistas, com posterior análise qualitativa de conteúdo, através de um processo sistemático de classificação e de codificação de temas ou padrões ${ }^{(16)}$.

Os resultados deste estudo (16) demonstram que os cuidadores familiares gerem a dependência da pessoa idosa com AVC na realização das AVD através de 
prioridades. Estas foram categorizadas em 7 estratégias: incentivo a movimentos físicos (treino de marcha precoce, diminuir a espasticidade e promover o equilíbrio), incentivo à higiene pessoal (banho, higiene oral, higiene após eliminação, lavagem das mãos), nutrição (a considerar: disfagia, SNG, hipertensão, diabetes), integração em atividades religiosas (a participação semanal promove a relação social e estabilidade conjugal), atividades de lazer (ouvir música, ver TV, ler, caminhar na rua), incentivo nas transferências (uso de auxiliar de marcha e adaptação do ambiente, diminuição do risco de queda) e assistência na gestão financeira (gestão da reforma mensal face as despesas, incluindo medicação).

Os autores ${ }^{(16)}$ concluíram que a família tem um papel fundamental na dependência da pessoa idosa com AVC, gerindo as suas AVD, nomeadamente no cuidado pessoal e mobilidade, bem como na parceria com os profissionais de saúde e ensinos de exercícios para a minimização daquela dependência. Assim, estes dados reforçam a importância da educação para a saúde no internamento e alta hospitalar garantindo uma gestão mais eficaz na dependência nas AVD.

O terceiro e último estudo ${ }^{(9)}$ integrado na nossa revisão sistemática da literatura, com nível de evidência 2.c (15), foi desenvolvido no Reino Unido em 2016, com 114 pessoas que foram admitidas numa unidade de neurorreabilitação e avaliados por uma equipa multidisciplinar. 0 estudo teve o objetivo de avaliar a relação em diferentes domínios cognitivos, nomeadamente na função executiva e o risco de queda ou compromisso do equilíbrio, numa amostra de adultos com doenças neurológicas no centro de reabilitação.

Para tal, foi realizado um estudo de coort prospetivo, com aplicação da escala de Berg e testes cognitivos, para avaliar a função, processamento da informação, memória verbal e visual, perceção visual e função intelectual. Os dados foram categorizados e analisados através da análise estatística pelo método de Poisson, entre a função cognitiva e risco de queda, através do Stata V12.

O estudo (9) destaca a importância das diferentes dimensões cognitivas, nomeadamente a função executiva no risco de queda, numa população de jovens adultos com alterações neurológicas, numa unidade de neurorreabilitação. O mesmo concluiu que na pessoa com AVC não existe relação direta entre o risco de queda e a função cognitiva, mas antes relação entre a função executiva, cognição e equilíbrio, o que pode ser explicado pela teoria do controle executivo sobre equilíbrio e marcha.

\section{DISCUSSÃO}

No dia-a-dia deparamo-nos com uma diversidade de situações que merecem a integração de forma consistente da evidência científica, nomeadamente a nível da viabilidade e pertinência da conceptualização, no processo de desenvolvimento de competências.

O regresso a casa é a base de uma vida satisfatória na comunidade. No entanto, para um regresso a casa seguro, deparamo-nos com muitos desafios no processo de transição. O plano de cuidados que envolve essa transição segura para a comunidade é a chave que permite uma reintegração com sucesso. Este processo é facilitado quando a equipa interdisciplinar é detentora de competências na área da comunicação, negociação, entrevista e conhecimento aprofundado sobre acessibilidade, estudo e prescrição de produtos de apoio e recursos disponíveis na comunidade para a reintegração, sendo neste enquadramento o EEER um elemento fundamental.

A avaliação neurológica é fundamental para o sucesso da reabilitação pois só assim é possível determinar o potencial de reabilitação, planeando as atividades e intervenções a realizar ${ }^{(3)}$ e ainda sinalizar e referenciar a pessoa. Para tal, a utilização de escalas e instrumentos de medida mensuráveis, objetivos, simples e não invasivos é um aspeto facilitador que permite avaliar a funcionalidade e diagnosticar as alterações que determinam limitações da atividade e incapacidade, elaborar e aplicar de planos de cuidados de enfermagem de reabilitação individualizados com formação e treino na sua implementação (3) (1).

\section{Dimensão motora, cognitiva e equilíbrio}

O treino motor é essencial para a recuperação da pessoa após AVC, melhorando a capacidade funcional, aumentando a qualidade de vida. A pessoa em situação de AVC e seus familiares consideram como objetivo principal da reabilitação, a capacidade de deambular, associada ao desenvolvimento das atividades de vida com exercícios motores, promotores do autocuidado. Os benefícios da atividade física após o AVC são muitos e variados como, por exemplo, aumento da aptidão cardiorrespiratória e diminuição de sintomas depressivos. Assim, neste sentido, foi desenvolvido um estudo (11) e aplicado um questionário que identifica múltiplas dimensões nas preferências de exercícios, revelando alta variabilidade individual na preferência nas condições do exercício, destacando a necessidade de exercícios pós-AVC sob aplicação de programas.

No entanto, o problema da pessoa com AVC que inicia um programa de exercício físico é manter o seu envolvimento a longo prazo. Os participantes com maior incapacidade identificam a necessidade de supervisão e apoio durante a realização dos exercícios em casa, o que contribuirá para que a autoconfiança e desafio estejam relacionados com a probabilidade de manter o exercício continuamente ${ }^{(8)}$.

Para a realização do exercício existem fatores que influenciam a segurança, nomeadamente o equilíbrio. Assim, a área cognitiva e a função executiva, mais especificamente, o raciocínio abstrato, o planeamento, a tomada de decisão, a inibição, a preservação e a impulsividade são afetadas e a recuperação a nível do equilíbrio é fortemente influenciada, existindo assim uma relação entre a cognição e o equilíbrio ${ }^{(9)}$. Com alterações a nível da cognição, também a comunicação e compreensão podem estar afetadas e, neste sentido, o recurso à comunicação verbal, nomeadamente a utilização de instruções ou feedback direcionando a atenção da pessoa sobre os movimentos que a pessoa está a realizar e efeitos dos mesmos, contribuem para 
uma melhoria da função motora, a nível da postura e reeducação da marcha ${ }^{(14)}$.

Os planos de cuidados de reabilitação ou mapa de exercícios com foco na mobilidade e AVD, com exercícios direcionados ao fortalecimento muscular, equilíbrio estático e dinâmico, como nos complementam outros autores ${ }^{(18)}$, permitem obter resultados significativos a nível do desenvolvimento do autocuidado, contribuindo para a prevenção de quedas e relação positiva significativa entre o tempo de terapia e o índice de Barthel. Para tal, devem ser fortemente envolvidos no processo de cuidados, para além da pessoa, os profissionais de saúde e a família, contribuindo para que todos tenham conhecimento da situação e evolução da pessoa, de acordo com os objetivos estabelecidos individualmente.

Neste sentido e ainda que os estudos analisados não mostrassem inequivocamente o envolvimento do EEER, a intervenção no âmbito da função muscular, atividades e exercícios terapêuticos, contribuem para aumentar a resistência, melhorar a força e a massa muscular, a amplitude de movimentos, o controlo da estabilidade postural e o equilíbrio e contribui também para a prevenção de quedas e outras complicações através do ensino e treino de AVD, capacitando a pessoa para o autocuidado e realização de exercício físico e automobilizações em segurança, em parceria com o cuidador ${ }^{(18)}$.

O ensino e treino do autocuidado proporciona a aquisição da máxima funcionalidade e qualidade de vida através de estratégias adaptativas e de produtos de apoio contribuindo para um regresso a casa e à comunidade. É nesta fase que se processa a integração da pessoa incapacitada nessa comunidade, que envolve para além do ambiente, disponibilidade de habitação, transportes, profissionais de saúde, localização geográfica, recursos e acesso a serviços locais (19) (20).

A música como atividade interdisciplinar de utilidade terapêutica no processo de reabilitação

0 potencial de reabilitação é influenciado pelo estado físico e psicológico da pessoa. As pessoas sofreram algo inesperado e naturalmente irão enfrentar um processo de ajustamento (negação, agressividade, depressão, reconhecimento, adaptação e aceitação) na gestão de emoções, na comunicação, gestão e valorização das conquistas. A insegurança está relacionada com a incapacidade funcional, depressão e fadiga, pelo que é sugerido que os programas de exercício envolvam também uma dimensão psicológica e emocional. As pessoas com sintomas depressivos apresentam baixos níveis de confiança, acabando por se isolar no domicílio (8). Assim, numa primeira fase, é necessário apoio e compreensão e posteriormente é essencial o encorajamento para a autonomia. Enquanto profissional e perito, é importante ter conhecimento dos objetivos e interesses da pessoa antes do evento, para poder gerir as expetativas, bem como transmitir a informação de modo claro, percetível e precoce.

A música pode assim ser um aliado neste processo para estimular as funções cerebrais envolvidas na cognição, fala, emoções, perceções sensoriais e, consequentemente, no movimento e autonomia. As intervenções musicais melhoraram significativamente a comunicação geral das pessoas com afasia, a marcha e a qualidade de vida da pessoa com AVC, bem como as suas habilidades sociais, principalmente quando associado o ritmo, em músicas da preferência da pessoa (21).

Neste sentido, a utilização da música pode assim ser uma experiência com benefícios demonstrados ${ }^{(19)}$, que pode ser desenvolvida ao ar livre, com recurso à música, a instrumentos musicais e à presença de vários profissionais, bem como de familiares. A sua prática deve englobar a combinação de música e movimentos, para melhorar a saúde física e psicológica durante o internamento na fase aguda da pessoa com AVC, através de educação para a saúde sobre o AVC e reabilitação, exercícios direcionados ao hemicorpo não afetado e, posteriormente, ao afetado, bem como à expressão de dificuldades e sentimentos ${ }^{(22)}$.

A pessoa, o cuidador e a equipa interdisciplinar na preparação do regresso a casa

A preparação do regresso a casa deve garantir a reabilitação precoce no seio da equipa de enfermagem, na capacitação de conhecimento, capacidades e habilidades para lidar com o bem-estar e autocuidado, promovendo a readaptação funcional da pessoa e envolvimento da família como parceira ativa onde existe cooperação e responsabilidade partilhada ${ }^{(3)}$.

A educação para saúde revela-se um eixo de investimento importante que contribui para a estabilização da funcionalidade e qualidade de vida, através da capacitação da pessoa e do cuidador. Como estratégias, o profissional pode recorrer a questionários sobre o conhecimento e necessidades da pessoa/cuidador, para posterior realização de reuniões e/ou sessões de educação para a saúde, criando momentos privilegiados para aquisição de competência e/ou partilha, compreensão, motivação com base de experiências pessoais e familiares ${ }^{(11)}$.

Tendo por base os dados que resultaram da aplicação do questionário ${ }^{(8)}$, das próprias pessoas na comunidade, é pertinente que os fatores e barreiras identificadas e relacionadas com a incapacidade física, ansiedade, depressão e fadiga sejam trabalhadas no internamento na preparação do regresso a casa, com o objetivo de serem minimizados, promovendo a adesão e a continuidade a longo prazo do programa de exercícios na comunidade.

Assim, o EEER deve garantir que a pessoa seja referenciada para a comunidade, assegurando apoio e supervisão na realização do programa de exercícios individualizado em contextos preferencialmente familiares e de amigos ou mesmo grupos comunitários, que integrem pessoas com idade e patologia semelhantes ${ }^{(8)}$.

O programa de exercícios deve ser mediado por cuidadores capacitados, com potencial para melhorar os resultados em termos de função corporal, atividades e participação em pessoas com AVC. Além disso, os 
cuidadores que estão envolvidos no processo de reabilitação, podem aumentar sentimentos de empoderamento com níveis reduzidos de excesso de carga no seu papel e facilitar a transição do hospital para a comunidade, diminuindo o tempo de internamento ${ }^{(23)}$.

A família tem um papel fundamental no eventual estado de dependência da pessoa idosa com AVC, através da gestão das suas AVD, bem como na parceria com os profissionais de saúde. Para tal, os cuidadores gerem a dependência nas atividades de vida através de prioridades, com especial atenção na mobilidade, com o treino de marcha precoce, transferência com ou sem uso de auxiliar de marcha e na higiene pessoal. Os familiares têm um papel fulcral nos ensinos dos exercícios para a manutenção da independência, na educação para a saúde no internamento e alta hospitalar com o objetivo de garantir uma melhor gestão na dependência nas atividades de vida (16).

A pessoa e família/cuidador capacitados para promover o regresso a casa devem demonstrar habilidades e competências na vigilância e controlo de fatores de risco, identificação e avaliação de sinais de alerta, assim como de sinais de agravamento e de complicações da doença e da promoção de um ambiente calmo e tranquilo propício ao processo de reabilitação tanto no internamento como no domicílio.

A promoção de relações familiares, sociais e comunitárias saudáveis, a continuidade de exercícios motores e atividades terapêuticas no domicílio, a promoção do desenvolvimento das AVD e atividades instrumentais de vida diária (AIVD) pela própria pessoa no seu tempo necessário, com as suas estratégias e em segurança, o ensino e treino de estratégias promotoras de autocuidado com recurso a equipamentos e produtos de apoio, contribuindo para a prevenção de quedas, a promoção das acessibilidades no domicílio e na comunidade e uma promoção efetiva da continuidade do processo de reabilitação, através do apoio e supervisão dos recursos da comunidade devem complementar este cenário.

Paralelamente ao processo de capacitação, não deve ser descurada uma avaliação do cuidador, nomeadamente dos seus medos, incapacidades e visão sobre o novo papel, compreender como vai ser o seu novo papel naquela família e estar alerta para situação de conflitos e exaustão do cuidador, garantindo a segurança dos cuidados $\mathrm{e}$ de todos os seus intervenientes ${ }^{(24)}$.

É na comunidade, no ambiente familiar, que se proporciona a continuidade dos cuidados necessários, na qual o acesso a serviços de saúde primários e assistência de reabilitação a pessoas com incapacidade pressupõem a utilização dos recursos aí localmente disponíveis, transferências de conhecimento, envolvimento no planeamento, tomada de decisão e avaliação e utilização e reforço dos serviços, no treino e supervisão ${ }^{(20)}$.

Sendo o regresso a casa um indicador de reabilitação de sucesso e, consequentemente, um indicador de qualidade, a prestação de cuidados de enfermagem de reabilitação deve ser realizada e, sempre que possível, em tempo útil, de acordo com as necessidades reais e potenciais, tendo em conta a individualidade de vida, promoção de um ambiente terapêutico e seguro, propício à tomada de decisão responsável.

Os programas de reabilitação assumem naturalmente um processo de transições, sendo fundamental o planeamento das intervenções de todos os profissionais de saúde integrados numa equipa interdisciplinar equilibrada (6) (7). No entanto, tem havido um aumento da necessidade dos cuidados de reabilitação paralelo ao aumento de pessoas com incapacidade por diferentes patologias e, para que este aspeto seja eficaz, é necessário um líder, moderador, pessoa de referência, responsável, designado de gestor de caso.

0 gestor de caso tem um papel imprescindível no processo interdisciplinar, devendo ser um profissional da equipa que assume a função de interlocutor único ou incontornável que, em reunião interdisciplinar apresenta uma síntese que valoriza as potencialidades da pessoa, os seus desejos e expetativas. Deve ter uma ação polivalente e flexível, aplicando as competências de um líder afetivo e especialista e ainda ser um elo cooperante, tanto no âmbito de gestor de caso, como gestor de cuidados e moderador na dinâmica do grupo (25). É responsável pela transição da pessoa para os diferentes recursos, de acordo com a suas necessidades, através de estratégias com objetivo de gerir os custos, readmissão e satisfação do cliente, garantindo a qualidade, segurança, eficiência, relação custo-benefício, justiça, igualdade, equidade, cuidados centrados na pessoa, promovendo a continuidade de cuidados ${ }^{(26)}$.

Estes aspetos reforçam naturalmente as competências do EEER, particularmente na prestação de cuidados, tendo por base a visão holística, contínua e sequencial na promoção da relação afetiva e de proximidade às pessoas e em que a intensidade do trabalho da equipa interdisciplinar procurará evidenciar melhoria funcional com a definição de objetivos direcionados para a tarefa, com benefícios previsíveis em dimensões como a força, o equilíbrio e as reaprendizagens relativas ao autocuidado.

A intervenção precoce, especializada e diária, para além de prevenir complicações e incapacidades, permite recuperar funções perdidas e/ou fortalecer funcionalidades similares às originais, uma vez que o cérebro tem uma grande capacidade de adaptação (3), sendo de extrema importância dar continuidade ao processo de reabilitação todos os dias, 24 horas por dia. Ao desenvolver um plano de alta, a pessoa pode ser referenciada para a Rede Nacional de Cuidados Continuados Integrados (RNCCl), de acordo com os respetivos critérios, que contempla apoio a pessoas em situação de dependência com necessidades de cuidados de saúde e de apoio social, de natureza preventiva, reabilitação ou paliativa, em unidades de internamento, unidade de ambulatório, equipas hospitalares e equipas domiciliárias ${ }^{(27)}$, em colaboração com a equipa de gestão de altas da instituição, tendo por base o percurso da pessoa/cuidador. 


\section{Implicações Práticas}

Tendo em conta o objetivo inicial e questão PICo: Que intervenções de Enfermagem de Reabilitação capacitam a pessoa e família/cuidador, em situação de AVC, com alteração da funcionalidade a nível motor, na preparação do regresso a casa?, devemos considerar que a Enfermagem de Reabilitação, tendo por base o seu regulamento de competências específicas ${ }^{(1)}$, tem um papel determinante na elaboração, implementação, avaliação e reformulação de programas de treino motor em função dos resultados esperados, que irão contribuir para a adesão e continuidade dos programas de reeducação funcional motora na comunidade, pela educação para a saúde e capacitação da pessoa com AVC e cuidador/família, através de uma abordagem psicológica, cognitiva e física desde a admissão, uma vez que o evento de AVC pressupõe internamento. Neste sentido, o EEER pode ser um elemento determinante na equipa interdisciplinar enquanto promotor de segurança e qualidade, com competências de excelência para o desenvolvimento de programas de melhoria contínua, integrando políticas de saúde que visem a adoção de estilos de vida saudáveis e favoráveis a um incremento da qualidade de vida.

\section{Limitações do Estudo}

$\mathrm{Na}$ revisão sistemática da literatura verificou-se escassez de artigos inequivocamente direcionados à questão PICo e dados específicos dos EEER, tendo em conta a realidade de outros países. No entanto, de acordo com os profissionais que englobam a equipa interdisciplinar e as competências específicas do EEER, foi possível identificar um conjunto de intervenções que fundamentam as atividades realizadas e o enquadramento teórico adotado. Assim, é possível afirmar que os artigos incluídos têm significado, sustentando a temática em estudo, dando resposta à questão PICo e fortalecendo a pertinência do EEER em todos os contextos na capacitação da pessoa e cuidadores na promoção do autocuidado, controlo da sua saúde e do ambiente que os envolve, quer seja a nível do internamento hospitalar em fase aguda, do internamento em unidades de reabilitação ou na comunidade, em equipas de suporte a essas pessoas e cuidadores.

\section{CONCLUSÃO}

A presente revisão sistemática da literatura tinha como objetivo responder à questão Que intervenções de Enfermagem de Reabilitação capacitam a pessoa $e$ família/cuidador, em situação de AVC, com alteração da funcionalidade a nível motor, na preparação do regresso a casa?, e com a análise dos artigos incluídos é possível concluir que os programas de reeducação funcional motora devem integrar a dimensão psicológica, cognitiva e física, a dependência e desenvolvimento de AVD, nomeadamente no cuidado pessoal e mobilidade, objetivos, expetativas e preferências da pessoa, orientação e supervisão garantindo adesão e continuidade a longo prazo o programa de reabilitação.
Neste sentido, o recurso à música pode ser uma estratégia para melhorar a saúde física e psicológica, a nível da cognição, fala, emoções, perceções sensoriais e, consequentemente, a autonomia e qualidade de vida.

O EEER, apesar de não ser referido explicitamente nos estudos, tem características essenciais e competências inerentes à função do gestor do caso na equipa interdisciplinar da pessoa com AVC, podendo garantir segurança na capacitação da familiar e/ou cuidador no processo de educação para a saúde, na mudança de fatores de risco modificáveis, contribuindo para a diminuição da incidência de eventos de AVC, bem como na continuidade do programa de reabilitação motora, com exercícios promotores de fortalecimento muscular e equilíbrio, promovendo a autonomia, qualidade de vida e transição segura para a vida na comunidade.

\section{REFERÊNCIAS BIBLIOGRÁFICAS}

1. Regulamento n. ${ }^{\circ} 392 / 2019$. Regulamento das Competências Específicas do Enfermeiro Especialista em Enfermagem de

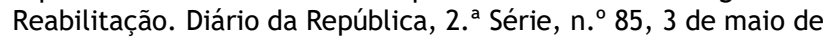
2019. Lisboa: Ordem dos Enfermeiros.

2. Meleis Al. Transitions theory middle-range and situation-specific theories in nursing research and practice. New York: Springer Publishing Company; 2010. Disponível em: https://taskurun.files.wordpress.com/2011/10/transitions_theo ry_middle_range_and_situation_specific_theories_in_nursing_r esearch_and_practice.pdf.

3. Menoita G, Sousa L, Alvo I, Vieira C. Reabilitar a pessoa idosa com AVC: Contributos para um envelhecer resiliente. Loures: Lusodidacta; 2014.

4. Direção-Geral da Saúde. Portugal: Doenças CérebroCardiovasculares em Números - 2015, Programa Nacional das Doenças Cérebro-Cardiovasculares. Lisboa: Direção Geral da Saúde; 2015. Disponível em: https://www.dgs.pt/emdestaque/portugal-doencascerebro-cardiovasculares-emnumeros-201511.aspx.

5. Everink I, Haastreght J, Hoof S, Scols J, Kempen G. Factores influencing home discharge after inpatient rehabilitation of old patients: a systematic review. BMCGeriatrics. 2016; 16:5. Disponível em: http: / / web.a.ebscohost.com/ehost/pdfviewer/pdfviewer?sid=0 6398018-b695-448da35abd2ba535d832\%40sessionmgr4008\&vid=20\&hid=4107.

6. Santos L. O Processo de Reabilitação. In Marques-Vieira C, Sousa L. Cuidados de Enfermagem de Reabilitação à Pessoa ao Longo da Vida (1 ${ }^{\mathrm{a}}$ ed). Loures: Lusodidacta; 2016: 15-24.

7. Branco PS. Equipa de Reabilitação. In Marques-Vieira C, Sousa L. Cuidados de Enfermagem de Reabilitação à Pessoa ao Longo da Vida ( $1^{\mathrm{a}}$ ed). Loures: Lusodidacta; 2016: 25-34.

8. Bonner NS, O'Halloran PD, Bernhardt J, Cumming TB. Developing the Stroke Exercise Preference Inventory (SEPI). Plos One. 2016; 11(10), e0164120:1-13. Disponível em: https://journals.plos.org/plosone/article?id=10.1371/journal.po ne.0164120.

9. Saverino A, Waller D, Rantell K, Parry R, Moriarty R, Playford ED. The role of cognitve factors in predicting balance and fall risk in a neuro-reabilitation setting. Plos One. 2016; 11(4), e0153469:1$14 . \quad$ Disponível em: https://www.ncbi.nlm.nih.gov/pubmed/27115880.

10. Sousa L, Marques-Vieira C, Branco, PS. Prevenir a Queda: Um Indicador de Qualidade dos Cuidados. In Marques-Vieira C, Sousa L. Cuidados de Enfermagem de Reabilitação à Pessoa ao Longo da Vida ( $1^{\mathrm{a}}$ ed). Loures: Lusodidacta; 2016: 559-570.

11. Cameron V. Best Practices for Stroke Patient and Family Education in the Acute Care Setting: A Literature Review. Medsurg Nursing. 2013; 22(1); jan-fev; 22(1): 51-5, 64. Disponível em:

http: //web.a.ebscohost.com/ehost/pdfviewer/pdfviewer?sid=0 
6398018-b695-448d-

a35abd2ba535d832\%40sessionmgr4008\&vid=16\&hid $=4107$.

12. Toubarro, F. Função Sensoriomotora. In Marques-Vieira C, Sousa L. Cuidados de Enfermagem de Reabilitação à Pessoa ao Longo da Vida ( $1^{\text {a }}$ ed). Loures: Lusodidacta; 2016: 159-166.

13. Veebeek JM, van Erwin W, van Roland P, Jan Yander W, Erik H, Rietberg M, Kwakkel G. What is the evidence for Physical Therapy Poststroke? A Systematic Review and Meta-Anlysis. Plos One. 2014; 9 (2), e87987:1- 33. Disponível em: https://www.ncbi.nlm.nih.gov/pmc/articles/PMC3913786/.

14. Matos MFG. A importância da Enfermagem de Reabilitação na preparação do regresso a casa da pessoa e família/cuidador em situação de AVC com alteração da funcionalidade motora. 2018. Mestrado em Enfermagem de Reabilitação. Santarém: Repositório Científico do Instituto Politécnico. Disponível em: https://repositorio.ipsantarem.pt/bitstream/10400.15/2252/1/ Relat\%c3\%b3rio\%20Completo\%20M\%20F\%c3\%a1tima\%20Matos.pdf.

15. Briggs J. Nursing: Evidence-Based Practice: Evidence Levels, Recommendations \& Types. Disponível em: https://guides.pnw.edu/evidence_based_practice/ebp_evidenc e.

16. Hesamzadeh A, Dalvandi A, Maddah SB, Khoshknab MF, Mosavi N. Family caregivers' experience of activities of daily living handling in older adult with stroke: a qualitative research in the Iranian context. Scandinavian Journal of Caring Sciences. 2017; 31:515 526. Disponível em: Disponível em: https://www.ncbi.nlm.nih.gov/pubmed/27530936.

17. Johnson L, Burridge JH, Demain SH. Internal and External Focus of Attention During Gait Re-Education: An Observational Study of Physical Therapist Practice in Stroke Rehabilitation. Physical Therapy. 2013; 93(7):957-966. Disponível em: https://www.ncbi.nlm.nih.gov/pubmed/23559523.

18. Huijben-Schoenmakers $M$, Rademaker A, Scherder E. 'Can practice undertaken by patients be increased simply through implementing agreed national guidelines?' An observational study. Clinical Rehabilitation. 2013; 27(6):513-250. Disponível em: https://www.ncbi.nlm.nih.gov/pubmed/23411792.

19. Ordem dos Enfermeiros: Mesa do Colégio da Especialidade de Enfermagem de Reabilitacão. Parecer MCEER n. ${ }^{\circ} 12 / 2011$ sobre as Atividades de Vida Diárias. 2011. Disponível em: http://www.ordemenfermeiros.pt/documentos/Documents/Par ecer12_MCEER_18_11_2 011_ActividadesVidaDiaria_AVD.pdf.

20. Hoeman, S. Enfermagem de Reabilitação - Prevenção, Intervenção e Resultados Esperados. $4^{\mathrm{a}}$ ed. Loures: Lusodidacta; 2011.

21. Magee WL, Clark I, Tamplin J, Bradt J. Music Interventions for acquired brain injury (Review). Cochrane Database of Systematic Reviews. 2017; issue 1. Art. No.:CD006787: 1-28. Disponível em: https: / /www.cochrane.org/CD006787/STROKE_musicinterventions-acquired-brain-injury.

22. Jun E, Young RH, Kim MJ. The effect of music-movement therapy on physical and psychological states of stroke patients. Journal of Clinical Nursing. 2013; 22:22-31. Disponível em: https://www.ncbi.nlm.nih.gov/pubmed/22978325.

23. Vloothuis JDM, Muder M, Veerbeek JM, Konijnenbelt M, VisserMeily JMA, Ket JCF, Kwakkel G, van Wegen EEH. Caregivermediated exercises for improving outcomes after stroke (Review). Cochrane Database of Systematic Reviews. 2016; issue 12. Art. No.:CD0011058: 1-113. Disponível em: https://www.cochranelibrary.com/cdsr/doi/10.1002/14651858. CD011058.pub2/full.

24. Martins, MM, Martins AC, Martins AR. Reeducação Familiar/Social - Reconstrução da Vida Familiar e Social no Processo de Reabilitação. In Marques-Vieira C, Sousa L. Cuidados de Enfermagem de Reabilitação à Pessoa ao Longo da Vida ( $1^{\text {a }}$ ed). Loures: Lusodidacta; 2016: 3-136.

25. Martins MCA, Fernandes PFC. O Gestor de Caso: aplicabilidade do conceito. 2010; disponivel http://hdl.handle.net/10400.11/1731.

26. Cesta T. Transitions in Care and the Role of Case Manager. Saúde e Medicina: Hospital Case Management: The Essencial Guide to Hospital-Based Care Planning. Atlanta. 2017; 25(12): 1-5. Disponível em: https://www.reliasmedia.com/articles/141685transitions-in-care-and-the-role-of-the-case-manager.

27. Portaria n. ${ }^{\circ} 174 / 2014$, de 10 de setembro. Diário da República, $1^{\text {a }}$ Série, n. ${ }^{\circ} 174,10$ de setembro de 2014. Ministérios da
Finanças, da Saúde e da Solidariedade, Emprego e Segurança Social. Lisboa. 